\title{
Predictive value of serology in diagnosing Lyme borreliosis
}

\author{
S J Cutler, D J M Wright
}

\begin{abstract}
Aims-To compare the predictive value of immunoblotting and enzyme linked immunosorbent assay (ELISA) in diagnosing Lyme borreliosis.

Methods-An ELISA using a whole cell sonicate of the B31 strain of Borrelia burgdorferi was used to screen samples submitted for Lyme borreliosis serology. A total of 1222 serum samples reactive in the ELISA were tested by immunoblotting also using the B31 strain. Patients with other spirochaetal diseases were tested by both methods to assess specificity, while those with erythema migrans were used to evaluate sensitivity. Subjects with different clinical conditions, which may have been associated with Lyme borreliosis, were tested using both techniques.
\end{abstract}

Results-Only $16 \cdot 3 \%$ of serum samples from patients submitted for Lyme borreliosis serology which were reactive by ELISA were confirmed as positive by immunoblotting. This is unlikely to represent a sensitivity problem as $51 \%$ of samples from 53 patients with erythema migrans were detected by immunoblotting compared with only $28 \%$ by ELISA. Patients whose samples were negative by ELISA were also negative by immunoblotting. Serum samples from patients with relapsing fever were reactive in both ELISA and by immunoblotting, but for other test groups immunoblotting offered increased specificity.

Conclusions-Not all ELISA results could be confirmed by immunoblotting. Yet immunoblotting was both more sensitive and specific than ELISA techniques. As a result of these observations all ELISA results should be serologically confirmed by immunoblotting. Though immunoblotting is not suited to large scale screening of samples, it can be used satisfactorily in conjunction with ELISA methods to improve the predictive value of serological tests for Lyme borreliosis.

Department of Medical Microbiology, Charing Cross

Hospital, London

IV6 8RF

S J Cutler

D J $M$ Wright

Correspondence to:

Dr Cutler

Accepted for publication

7 October 1993
(7 (Clin Puthol 1994;47:344-349)

Scrology remains by necessity the preferred method of diagnosing Lyme borreliosis in a laboratory. Enzyme linked immunosorbent assay (ELISA) has been used for serological screening, but this technique is plagued by problems of specificity. Improvements in the specificity of ELISAs have been explored by using either purified antigen such as the 41 kilodalton flagellin protein ${ }^{1}$ or semipurified preparations of Borrelia burgdorferi. ${ }^{2}$ Although these second generation ELISAs can reduce the number of serum samples giving false positive results, they do not entirely eliminate the problem.

Serological reactivity can be confirmed by immunoblotting. As the disease progresses, patients produce antibodies against an increasing range of both specific and nonspecific borrelial proteins. The interpretation of immunoblot patterns is controversial. Some studies have reported poor specificity of immunoblotting, ${ }^{3}$ while others maintain that the technique is highly specific. ${ }^{45}$ We devised an immunoblotting method to test samples submitted for Lyme borreliosis serology to our reference laboratory and evaluated this with serum samples from patients with Lyme borreliosis, healthy controls, and patients with other disorders.

\section{Methods}

Serum samples submitted for Lyme borreliosis serology were initially tested with an ELISA. A total of 1222 samples submitted over four years gave positive values on ELISA and were further tested by immunoblotting. In addition, samples from 53 patients with erythema migrans diagnosed by a dermatologist served as definite clinical cases of Lyme borreliosis. We also had samples from forestry workers $(n=305)$ from various parts of Britain, who had a high exposure to ticks.

Fifty consecutive samples submitted for serological testing that had negative results on ELISA were tested by immunoblotting to see whether immunoblotting was a worthwhile routine procedure. In addition, 50 samples submitted for routine antenatal serology and 65 samples from healthy subjects that had raised values on ELISA (from an initial group of 1139) served as negative controls.

To compare the specificity of the tests serum samples from subjects with other spirochaetal diseases were studied (louse borne relapsing fever (81 samples from 25 patients); syphilis (20 samples); and samples submitted for Lyme disease serology that were positive for syphilis (25 samples from 24 patients)).

Samples from patients with clinical conditions possibly associated with late borrelial infection and reactive in the ELISA screening test were also tested by immunoblotting. 
These included those from patients with dilated cardiomyopathy or myocarditis (N R B Cary et al, fourth international conference on Lyme borreliosis, Stockholm, 1990) (18 positive on ELISA from a study group of 282 patients); scleroderma (24 from a series of 175 patients); and chronic fatigue (five patients from an initial test group of 85 ).

\section{SEROLOGICAL METHODS}

The ELISA used to screen samples and the immunoblotting technique have been described in detail elsewhere ${ }^{67}$ Briefly, wells of an ELISA microtitre plate were coated with sonicated whole cells of $B$ burgdorferi (B31 strain) in carbonate buffer. After overnight incubation at $4^{\circ} \mathrm{C}$ the plates were washed three times and then incubated at $37^{\circ} \mathrm{C}$ for an hour sequentially with a blocking solution, diluted test serum ( 1 in 300), and a conjugate of goat antibody to human IgG with horseradish peroxidase; the final incubation was with o-phenylenediamine substrate for half an hour at room temperature before absorbance was read at $492 \mathrm{~nm}$. Absorbance was converted into arbitrary ELISA units, with those above the 95th percentile for healthy control subjects being recorded as weakly positive and those greater than the 98th percentile as positive.

Immunoblotting also used the B31 strain of $B$ burgdorferi, which was separated by discontinuous sodium dodecyl sulphate with polyacrylamide gel electrophoresis with $12.5 \%$ gels and transferred to nitrocellulose. Sheets of nitrocellulose were blocked overnight with dried milk powder and then washed and cut into strips. These strips were reacted with 1 in 200 dilutions of the patient's serum overnight and then washed, and incubated with goat antibody to human IgG conjugated with alkaline phosphatase. Bands were detected using p-nitroblue tetrazolium chloride and 5bromo-4-chloro-3-indolyl phosphate p-toluidine salt substrate.

Interpretation of $\mathrm{IgG}$ banding patterns was based on comparing the immunoblot profiles of healthy subjects with the profiles of patients with clinical Lyme borreliosis. This led to the development of a subjective system. Bands of 93, $31($ OspA), 30 (OspA leading front), and 20 kilodaltons were found in patients with Lyme borreliosis and considered to be specific; bands of $82,58-56$ doublet, 52,50 , 48,39 , and 34 (OspB) were often seen but were occasionally found in samples from patients with other disorders and thus only considered to be semispecific; and, finally, bands including $76,66,62,60,41$, and 15 kilodaltons did not correlate with disease. Typically healthy subjects had bands at 41 and 60 kilodaltons. This method was later adapted with numerical values assigned to reflect the relative importance of immunoblot bands. ${ }^{7}$

Additional strains of Lyme borreliosis spirochaetes were tested by immunoblotting using samples from patients who had acquired their illness in Europe (6), the United States (9), or the United Kingdom (11). These sam- ples were tested in parallel with the B31 strain of $B$ burgdorferi, together with the Swedish ACA isolate (ACA1), American human blood isolate (HB19), American CSF strain (297), and United Kingdom tick isolate (UK).

\section{Results}

Immunoblotting of 50 antenatal serum samples from London failed to produce any positive results. Typically, these samples only reacted to a band of 76 kilodaltons (nine), the 60 kilodaltons common antigen (21), 41 kilodalton flagellum protein of $B$ burgdorferi (36). Similarly, samples from 50 consecutive patients submitted for Lyme borreliosis serology that had given negative results on ELISA were all negative by immunoblotting. Patients with established Lyme borreliosis usually showed reactivity to a much wider range of proteins.

When the results of ELISA and immunoblotting were compared in the samples submitted for Lyme borreliosis serology that had given positive results on ELISA only 199 of $1222(16 \cdot 3 \%)$ samples were positive by immunoblotting (table 1). When weakly reactive serum samples were removed the proportion of ELISA results positive by immunoblotting rose only to $24 \cdot 4 \%$.

Geographical differences were seen in the United Kingdom. The proportion of samples from forestry workers in Scotland that could be confirmed by immunoblotting was smaller than the proportion from similar groups in England (table 2).

No difference in immunoblot interpretation as positive, equivocal, or negative was observed in samples from patients giving travel histories in America and Europe, or those who had not left Britain, when tested using either the American B31 reference strain, 297, or HB19, the Swedish skin isolate ACA1, or a British tick isolate of B burgdorferi, although minor differences in banding profiles were seen-for example, in possession of a 39 kilodalton band in the first of the British patients illustrated in the figure.

Table 1 Results on immunoblotting in 1222 samples positive by ELISA. Values are numbers (percentages) of samples

\begin{tabular}{lcc}
\hline \multirow{2}{*}{ ELISA result } & \multicolumn{2}{l}{ Immunoblotting result } \\
\cline { 2 - 3 } & Negative & Positive \\
\hline Weakly positive $(\mathrm{n}=608)$ & $559(91 \cdot 9)$ & $49(8 \cdot 1)$ \\
Strongly positive $(\mathrm{n}=614)$ & $464(75 \cdot 6)$ & $150(24 \cdot 4)$ \\
Total $(\mathrm{n}=1222)$ & $1023(83 \cdot 7)$ & $199(16 \cdot 3)$ \\
\hline
\end{tabular}

Table 2 Positive results on ELISA and immunoblotting in British forestry workers

\begin{tabular}{lccc}
\hline Group & $\begin{array}{l}\text { No of } \\
\text { workers }\end{array}$ & $\begin{array}{l}\text { No (\%) } \\
\text { ELISA }\end{array}$ & $\begin{array}{l}\text { No (\%) on } \\
\text { immunoblotting }\end{array}$ \\
\hline New Forest rangers & 42 & $12(28 \cdot 6)$ & $5(11 \cdot 9)$ \\
Thetford Forest & 78 & $16(20 \cdot 5)$ & $11(14 \cdot 1)$ \\
Scottish foresters & 142 & $23(16 \cdot 2)$ & $2(1 \cdot 4)$ \\
Scottish deer workers & 43 & $7(16 \cdot 3)$ & $2(4 \cdot 6)$ \\
Total & 305 & $58(19)$ & $20(6 \cdot 6)$ \\
\hline
\end{tabular}


When individual serological tests were evaluated with known cases of erythema migrans authenticated by a dermatologist, serology at its best could only detect half of the cases (table 3). Lowering the diagnostic cut off points of the ELISA would not resolve this problem as no significant difference could be detected when absorbance values from 51 patients with erythema migrans were compared with those from 120 healthy blood donors (Mann-Whitney $U$ test, $p=0 \cdot 498$, data not shown).
Reactivity of British patients (A), European patients (B), and American patients $(C)$ with Lyme borreliosis strains isolated from America, Europe, and Britain.

Lane 1 = tick strain B31; lane 2 = Swedish ACA1; lane $3=$ human blood isolate HB19;

lane $4=C S F$ isolate 297; and lane $5=U K$ tick strain.

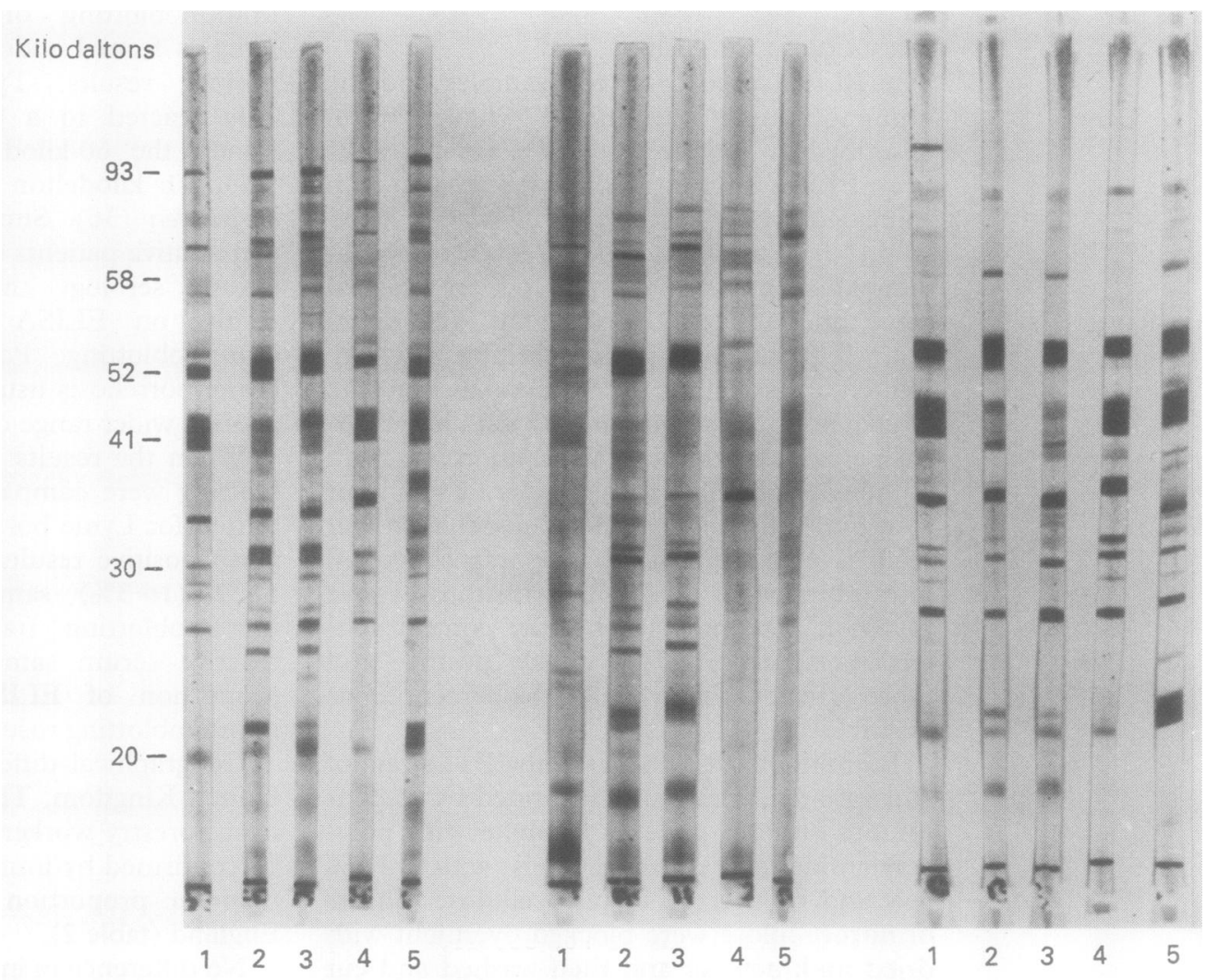

(A)

British cases

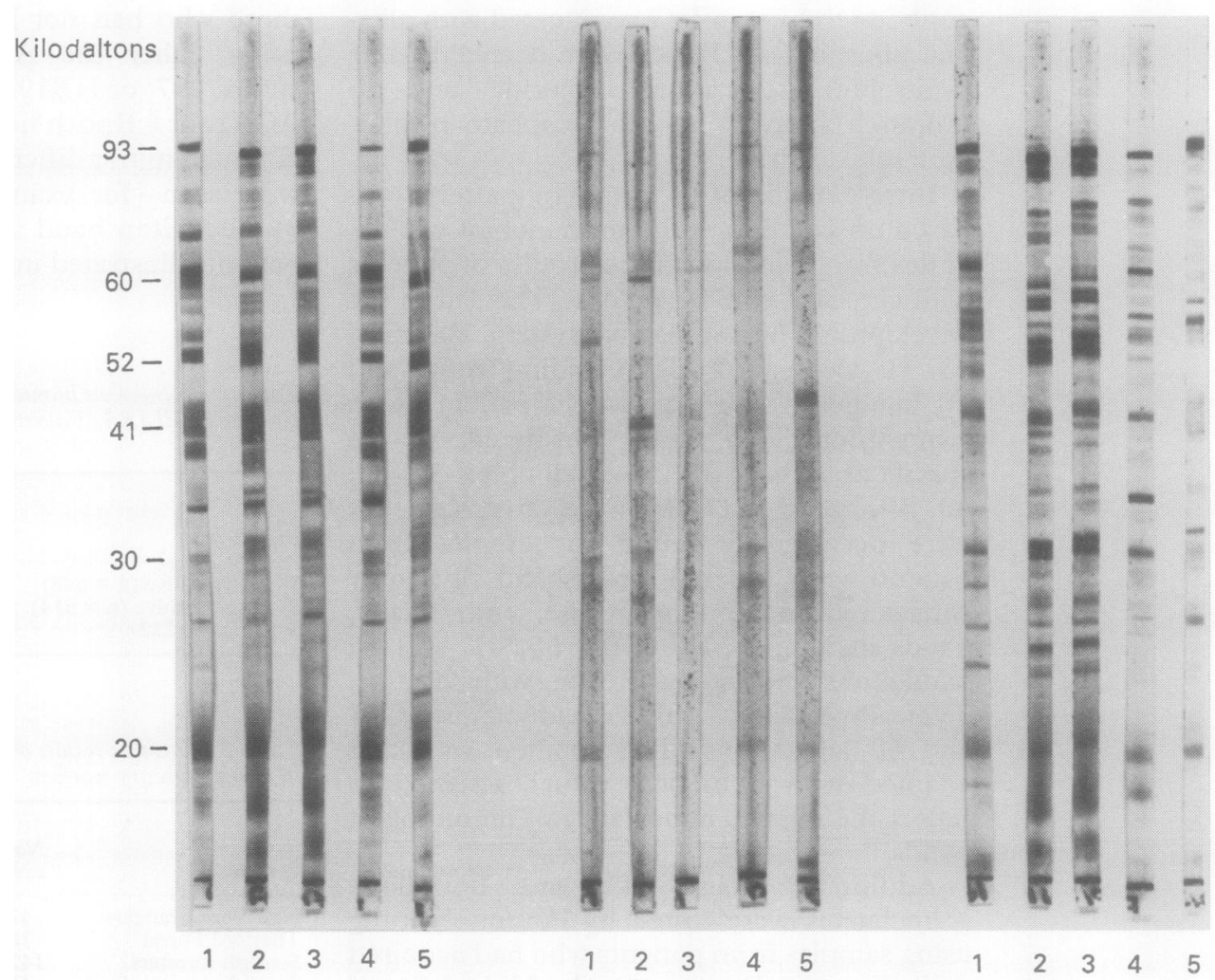

(B)

European cases 


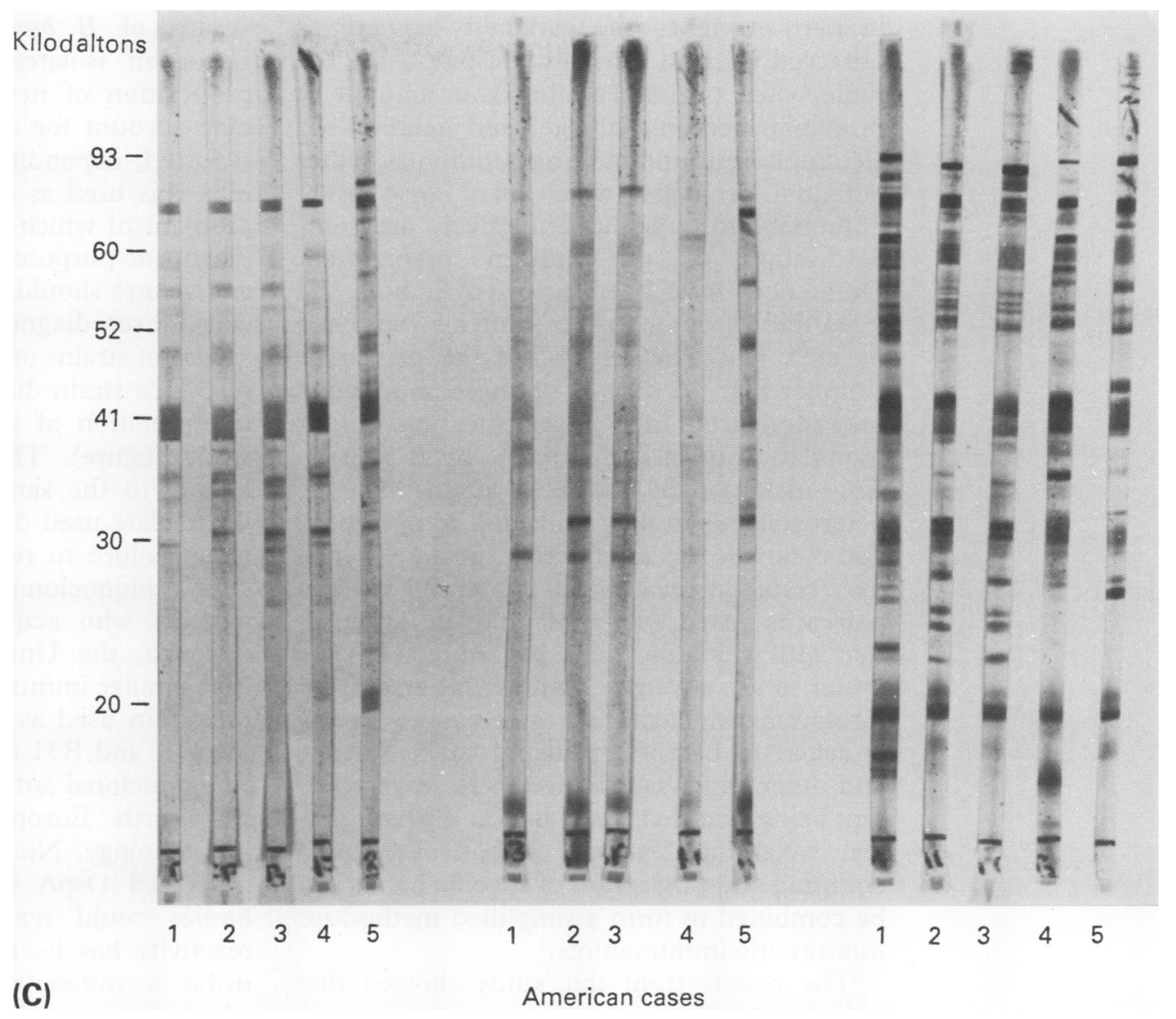

Table 3 Comparison of positive results in serological tests in 53 patients with erythema migrans

\begin{tabular}{ll}
\hline Serological test & No (\%) of patients seropositive \\
\hline ELISA & $15(28)$ \\
Immunoblotting & $27(51)$ \\
\hline
\end{tabular}

samples from five patients with chronic fatigue that had been positive by ELISA could be confirmed by immunoblotting.

\section{Discussion}

A major problem encountered with serological tests for Lyme borreliosis is the lack of specificity. Patients with a variety of other spirochaetal diseases and unrelated clinical conditions have been reported to give false positive serological results. Samples may cross react by binding to non-specific antigens of $B$ burgdorferi such as the 60 kilodalton common antigen or heat shock related proteins. ${ }^{910}$ Immunoblotting has been regarded as a "gold standard" method for laboratory confirmation of Lyme borreliosis. ${ }^{4}$ As the disease progresses antibodies are produced against a range of borrelial proteins, both specific and non-specific. The interpretation of immunoblot patterns is controversial, with many different schemes available. Some studies have reported poor specificity of immunoblotting, ${ }^{3}$ while others maintain that the technique is highly specific. ${ }^{4}$ These discrepancies largely result from differing methods used for interpretation. The principal methods used for the interpretation of immunoblots are those based on band counting, which includes both specific and non-specific proteins, ${ }^{4}$ or those which require possession of selected bands such as a single band of $18,21 \cdot 5$, or 23 kilodaltons in conjunction with the 41 kilodalton flagellum. ${ }^{11}$ Some studies have proposed that quantification of bands may be valuable in interpreting immunoblot bands. ${ }^{5}$ This type of interpretation was not evaluated in this study, 
in part owing to the variability sometimes observed in band intensity, despite using the same batch of antigen, the same amount of protein loaded on to the gel, and standardised electrophoretic and blotting conditions. Other potential problems which may arise using immunoblotting semiquantitatively are standardisation of the antigen preparation. Differences have been reported in both the quantities of outer surface proteins expressed by cells and whether or not the protein is expressed at all. Some of these differences may result from in vitro selective pressure, or modulation or loss of antigens by $B$ burgdorferi. ${ }^{12}$ Additionally, borrelial strains may be heterogeneous in their antigenic composition, and comprise several different species, ${ }^{13}$ which may result in greater band intensity when a patient is tested with a homologous strain to that with which they have been infected. ${ }^{11} \mathrm{We}$ prefer to use a weighted numerical system for interpretation, with greater value being attached to bands considered to be specific and other bands considered to be less important being awarded lesser values. With numerical weighting features of both the band counting and possession of specific bands can be combined to form a simplified method for interpreting immunoblots. ${ }^{7}$

The results from this study showed that only $16.3 \%$ of samples that were positive on ELISA could be confirmed as genuinely positive by immunoblotting. Therefore, what do the remaining samples with positive results on ELISA and negative results on immunoblotting represent? These may illustrate a specificity problem of the ELISA, with patients producing antibody against non-specific borrelial proteins. As the ELISA utilises 95th and 98th percentiles of healthy individuals for its test cut off values, it follows that $2 \%$ of well ambulatory subjects will be strongly seropositive and $3 \%$ weakly seropositive. When ELISA was used to test 1139 healthy individuals from various geographical locations in the United Kingdom, $65(5 \cdot 7 \%)$ gave positive ELISA values. None of these individuals could be confirmed as having Lyme borreliosis by immunoblotting.

Alternatively, patients with positive results on ELISA but negative results on immunoblotting may not have had the disease sufficiently long to produce a positive pattern on immunoblotting. Not all patients may be able to produce an immune response to diagnostic bands and early antibiotic treatment may prevent a positive immunoblot pattern developing. In addition, the diversity of borrelial strains causing Lyme borreliosis may result in false negative immunoblots if a different strain or species is used as antigen.

The ELISA used in this study gave comparable findings with other ELISAs for Lyme borreliosis that are available commercially (data not shown). ${ }^{14}$ Second generation ELISAs still showed cross reactivity with patients with other spirochaetal diseases and produced positive findings which could not be confirmed by immunoblotting.

Considerable diversity exists between strains of $B$ burgdorferi, especially among European isolates, resulting in the recent proposition of new genomic species. ${ }^{13}$ This may account for differences in seropositivity reported, depending on which strain of borrelia was used as antigen, and highlights the problem of which isolate should be used for diagnostic purposes. Possibly a panel of different isolates should be used for preparing antigens for serodiagnosis?

Use of strains other than the American B31 reference strain did not significantly alter the interpretation of immunoblot results in this study (figure). The strains tested might all belong to the same borrelial species. ${ }^{13}$ The UK isolate used differed from the B31 strain in its failure to react with the "species specific" monoclonal antibody H5332, but patients who acquired Lyme borreliosis in America, the United Kingdom, or Europe gave similar immunoblot results regardless of the strain used as antigen (figure). Although the UK and B31 strains differed in reactivity to monoclonal antibody H5332 (S J Cutler et al, fourth European congress of clinical microbiology, Nice, 1989), both strains still possessed OspA with which patients' antibodies could react. Different immunoblot reactivity has been reported by Wilske et al using a different strain as antigen, ${ }^{15}$ but whether this would have caused results to be erroneously recorded as positive or negative was not disclosed.

Immunoblotting in this study was more sensitive than ELISA, detecting $50.9 \%$ of patients with erythema migrans compared with the $28.3 \%$ found by ELISA. Specificity was also superior using immunoblotting; none of the 65 samples that were positive on ELISA from healthy subjects gave a positive result on immunoblotting and only one of the 45 samples that were positive on ELISA from patients with syphilis gave a positive result on immunoblotting. The 81 samples from patients with relapsing fever were highly cross reactive in both ELISA and immunoblot analysis, with 49 and 45 respectively giving falsely positive results.

Patients with cardiac disease, scleroderma, or chronic fatigue, which may have been associated with chronic borrelial infection and which had given positive ELISA results, had essentially negative results, suggesting that Borrelia sp does not cause these conditions.

Problems common to all serological tests are that the IgG response to $B$ burgdorferi rises slowly, often not achieving significant concentrations until the sixth week after infection. Titres of IgG can remain raised years after clinical remission or after asymptomatic exposure, ${ }^{16}$ which complicates diagnosis in patients with unrelated clinical presentation. Additionally, some patients with clinical Lyme borreliosis fail to mount a clinically significant antibody response. This may result from prompt treatment which has been reported to abrogate the antibody response. ${ }^{17}$ Alternatively, antibody production may be restricted to the site of active disease-for example, in neuroborreliosis the cerebrospinal 
fluid may give positive results while serum gives negative results. ${ }^{18}$ Antibody may remain sequestered in immune complexes and therefore not detectable by commonly used assay methods. Cases have been reported with no significant $B$ cell response, although specific $T$ cell proliferative responses were detected. ${ }^{19}$ The humoral response, unlike the cellular immune response, may be selectively suppressed during early borreliosis. ${ }^{20}$

Immunoblotting was more sensitive than ELISA in detecting disease in patients with early Lyme borreliosis as well as more specific: the only cross reaction was with samples from subjects with relapsing fever. This technique, however, is not readily automated and is too expensive to use as a screening method for serodiagnosis of Lyme borreliosis. Alternatively, use of ELISA alone would result in a large number of false positive results. Although some improvement is offered with second generation ELISAs, specificity is still a problem. ELISA is best suited to screen for samples giving negative results, while immunoblotting should be reserved for confirming serological reactivity and for cases with a high index of clinical suspicion.

1 Hansen K, Hindersson P, Pedersen NS. Measurement of antibodies to the Borrelia burgdorferi flagellum improves s6:338-46.

2 Magnarelli LA, Anderson JF, Barbour AG. Enzyme-linked immunosorbent assays for Lyme disease: reactivity of subunits of Borrelia burgdorferi. F Infect Dis 1989;159. 43-9.

3 Golightly MG, Viciana AL. ELISA and immunoblots in the diagnosis of Lyme borreliosis: sensitivities and sources of false-positive results. In: Schutzer SE ed. sources of false-positive results. In: Schutzer SE, ed. Current communications in cell and molecular biology. Vol 6.
Cold Spring Harbor: Cold Spring Harbor Laboratory Press, 1992:283-97.

4 Grodzicki AL, Steere AC. Comparison of immunoblotting and indirect enzyme-linked immunosorbent assay using different antigen preparations for diagnosing early Lyme disease. F Infect Dis 1988;157:790-7.

5 Zöller L, Burkard S, Schäfer H. Validity of Western immunoblot band patterns in the serodiagnosis of Lyme borreliosis. f Clin Microbiol 1991;29:174-82.

6 Cutler SJ, Wright DJM. Comparison of immunofluorescence and enzyme linked immunosorbent assays for diagnosing Lyme disease. 7 Clin Pathol 1989;42:869-71.

7 Cutler SJ, Wright DJM, Luckhurst VH. Simplified method for the interpretation of immunoblots for Lyme borreliofor the interpretation of immunoblots for Lyme borreliosis. Federation of European Microbiological Socie

8 Magnarelli LA, Anderson JF, Johnson RC. Cross-reactivity in serological tests for Lyme disease and other spirochetal infections. F Infect Dis 1987;156:183-8.

9 Carreiro MM, Laux DC, Nelson DR. Characterization of the heat shock response and identification of heat shock protein antigens of Borrelia burgdorferi. Infect Immun 1990;58:2186-91.

10 Hansen K, Bangsborg JAM, Fjorduang H, Petersen NO Hindersson P. Immunochemical characterization of and isolation of the gene for a Borrelia burgdorferi immunodominant 60 kilodalton antigen common to a wide range of bacteria. Infect Immun 1988;56:2047-53.

11 Karlsson $M$, Hovind-Hougen $K$, Svenungsson $B$ Stiernstedt G. Cultivation and characterization of spiroStiernstedt G. Cultivation and characterization of spiro-
chetes from cerebrospinal fluid of patients with Lyme borreliosis. F Clin Microbiol 1990;28:473-9.

12 Schwan TG, Burgdorfer W, Garon CF. Changes in infectivity and plasmid profile of the Lyme disease spirochete Borrelia burgdorferi, as a result of in vitro cultivation. Infect Immun 1988;56:1831-6.

13 Baranton G, Postic D, Saint Girons I, Boerlin P, Piffaretti $\mathrm{J}-\mathrm{C}$, Assous $\mathrm{M}$, et al. Delineation of Borrelia burgdorferi sensu stricto, Borrelia garinii sp nov and group VS461 associated with Lyme borreliosis. Int $¥$ Systems Bacteriol 1992;42:378-83.

14 Cutler SJ. Serological surveillance of Lyme borreliosis in the United Kingdom (1986-1990) [PhD thesis] London: University of London, 1992: appendix 2: 324-42.

15 Wilske $B$, Preac-Mursic V, Fuchs $R$, Bruckbauer $H$ Hofmann A, Zumstein G, et al. Immunodominant proteins of Borrelia burgdorferi: implications for improvin serodiagnosis of Lyme borreliosis. In: Neu HC, ed. Frontiers of infectious disease: new antibacterial strategies. Edinburgh: Churchill Livingstone, 1990:47-63.

16 Fahrer H, van der Linden SM, Sauvain M-J, Gern L Zhioua E, Aeschlimann A. The prevalence and incidence of clinical and asymptomatic Lyme borreliosis in dence of clinical and asymptomatic Lyme borreliosi

17 Preac-Mursic V, Weber K, Pfister H-W, Wilske B, Gross B, Baumann A, et al. Survival of Borrelia burgdorferi in antibiotically treated patients with Lyme borreliosis Infection 1989;17:355-9.

18 Baig S, Olsson T, Link H. Predominance of Borrelia burgdorferi specific $\mathbf{B}$ cells in cerebrospinal fluid in neuroborreliosis. Lancet 1989;334:71-4.

19 Dattwyler RJ, Volkman DJ, Luft BL, et al. Seronegative Lyme disease. Dissociation of specific T- and B-lymphocyte responses to Borrelia burgdorferi. $N$ Engl $7 \mathrm{Med}$ 1988;319:1441-6.

20 Coyle PK, Deng Z, Krupp LB, Belman AL, Benach JL Luft BJ. Detection of Borrelia burgdorferi antigen in cerebrospinal fluid from neurological Lyme disease patients in the absence of anti-B burgdorferi antibodies. Ann Neurol 1992;32:295 (abstract). 